\title{
ЛЕМНИСКАТА И НЕРАВЕНСТВА ДЛЯ ЛОГАРИФМИЧЕСКОЙ ЕМКОСТИ КОНТИНУУМА
}

\section{В. Н. Дубинин}

Показано, что для любого полинома $P(z)=z^{n}+\cdots$ со связной лемнискатой $E(P)=\{z:|P(z)| \leqslant 1\}$ и с $m$ критическими точками, и для любых $n-m+$ 1 точек лемнискаты $E(P)$ существует континуум $\gamma \subset E(P)$ логарифмической емкости сар $\gamma \leqslant 2^{-1 / n}$, соединяющий эти точки, а также все нули и критические точки полинома $P$. В качестве следствий приводятся некоторые оценки для континуумов наименьшей емкости, содержащих наперед заданные точки.

Библиография: 7 названий.

1. Введение и формулировка основного результата. Всюду ниже

$$
P(z)=\prod_{\mu=1}^{n}\left(z-z_{\mu}\right),
$$

где $n \geqslant 2$ и $z_{\mu}, \mu=1, \ldots, n,-$ комплексные числа. Множество

$$
E(P)=\{z:|P(z)| \leqslant 1\}
$$

принято называть лемнискатой [1]. Метрическим свойствам лемнискаты, а также взаимному расположению нулей $z_{\mu}$ и критических точек полинома $P$ (т.е. точек, где $\left.P^{\prime}=0\right)$ посвящено немало работ, а некоторые задачи в данной тематике не решены до сих пор (см., например, [1], [2]). Весьма полезным при изучении свойств лемнискаты является понятие логарифмической емкости континуума (далее - емкости) [3]. В данной заметке получено емкостное неравенство, характеризующее в некоторой степени разброс нулей и критических точек полинома $P$ внутри лемнискаты $E(P)$. С другой стороны, если лемниската известна, то это неравенство можно трактовать как оценку емкости континуума, проходящего через заданные точки. Предположим, что

$$
\max \left\{|P(\zeta)|: P^{\prime}(\zeta)=0\right\} \leqslant 1 .
$$

Тогда множество $E(P)$ связно, и поэтому следующее утверждение верно с константой $\sigma=1$. Для любых точек $a$ и $b$ лемнискаты $E(P)$ существует континуум $\gamma \subset E(P)$ емкости сар $\gamma \leqslant \sigma$, содержащий точки $a, b$, а также все нули и все критические точки полинома $P$. В роли $\gamma$ в этом случае выступает само множество $E(P)$. Естественно поставить вопрос о наименьшем значении величины $\sigma$, при котором указанное выше утверждение имеет место для любых полиномов (1). Заметим, что условие (2) в такой постановке является необходимым, ибо в противном случае множество $E(P)$ несвязно. Справедлива следующая

Работа выполнена при финансовой поддержке Российского фонда фундаментальных исследований, грант № 05-01-00099.

(C) В. Н. Дувинин, 2006 
ТеОРемА. Пусть полином (1) удовлетворяет условию (2), и пусть $m$ - число всех критических точек этого полинома. Тогда для любых $n-m+1$ точек лемнискаты $E(P)$ существует континуум $\gamma \subset E(P)$ емкости

$$
\operatorname{cap} \gamma \leqslant 2^{-1 / n}
$$

содержащий эти точки, а также все нули и критические точки полинома $P$.

Число "свободных" точек лемнискаты $E(P)$ в оценке $(3)$ равно $n-m+1 \geqslant 2$. Поэтому независимо от числа критических точек для любых двух точек лемнискаты всегда найдется континуум $\gamma \subset E(P)$, удовлетворяющий (3) и соединяющий эти точки с нулями и критическими точками полинома $P$. Пример полинома $P(z)=$ $T_{n}\left(2^{(1-n) / n} z\right)$, где $T_{n}(z)=2^{n-1} z^{n}+\cdots-$ полином Чебышева, двух точек $\pm 2^{1-1 / n}$ и континуума $\gamma=\left[-2^{1-1 / n}, 2^{1-1 / n}\right]$, показывает, что постоянная в правой части неравенства (3) не может быть улучшена. Наибольшее число "свободных" точек равно $n$, и в этом случае необходимо $m=1$, а $P(z)=\left(z+d_{1}\right)^{n}+d_{2}$, где $d_{1}$, $d_{2}-$ комплексные числа. Полагая для определенности $d_{1}=0, d_{2}=-1$, легко заключаем, что равенство в (3) достигается для полинома $P(z)=z^{n}-1$, точек $\sqrt[n]{2} \exp (2 \pi i j / n)$, $j=1, \ldots, n$, и континуума $\gamma=\left\{z:|z| \leqslant \sqrt[n]{2}, \arg z^{n}=0\right\}$ (о вычислении емкости см. $[3 ;$ с. 215,216$])$. Этими примерами не исчерпываются все случаи равенства в $(3)$. Отметим, что из неравенства (3) вытекает известная оценка сверху для диаметра лемнискаты $E(P)$ в случае, когда полином $P$ удовлетворяет условию $(2)$ :

$$
\operatorname{diam} E(P) \leqslant 2^{2-1 / n}
$$

[4]. В заключительной части данной статьи рассматриваются также некоторые оценки для контиуумов наименьшей емкости, вытекающие из неравенства (3).

2. Доказательство теоремы. Обозначим через $\zeta_{\nu}, \nu=1, \ldots, m$, все различные критические точки полинома $P$, и пусть $a_{j}, j=1, \ldots, n-m+1,-$ произвольные точки лемнискаты $E(P)$ из условия теоремы. Пусть $\mathscr{P}$ - аналитическая функция, обратная функции $w=P(z)$, и пусть $\mathscr{R}-$ риманова поверхность функции $\mathscr{P}[5$; гл. 10]. Далее рассматриваем функцию $\mathscr{P}$ как однозначную функцию, заданную на поверхности $\mathscr{R}$. Под проекцией точки $W \in \mathscr{R}$ понимается точка $P(\mathscr{P}(W)) \in \overline{\mathbb{C}}_{w}$. Отрезком на $\mathscr{R}$ назовем любую жорданову кривую на поверхности $\mathscr{R}$, однолистно лежащую над некоторым отрезком в $w$-плоскости. Аналогично определяется луч на $\mathscr{R}$ как жорданова кривая, однолистно лежащая над некоторым лучом вида $\arg w=$ const, $\rho \leqslant|w| \leqslant \infty$. Обозначим через $\mathscr{S}$ объединение всех отрезков на $\mathscr{R}$, у которых начало расположено над точкой $w=0$, а конец совпадает с конечной точкой ветвления поверхности $\mathscr{R}$ (т.е. с одной из точек $\left.\mathscr{P}^{-1}\left(\zeta_{\nu}\right), \nu=1, \ldots, m\right)$. В случае нулевых критических значений некоторые из этих отрезков могут оказаться вырожденными. Покажем, что множество $\mathscr{S}$ связно и каждая точка поверхности $\mathscr{R}$, лежащая над $w=0$, принадлежит $\mathscr{S}$. Для этого разобьем поверхность $\mathscr{R}$ лучами, соединяющими конечные точки ветвления $\mathscr{R}$ с бесконечностью, на $n$ простых листов (далее - листов) $L_{k}, k=1, \ldots, n$. Каждый лист $L_{k}$ суть плоскость с одним или, возможно, большим числом разрезов, $k=1, \ldots, n$. Поэтому каждый лист $L_{k}$ содержит, по крайней мере, один отрезок (возможно вырожденный), принадлежащий $\mathscr{S} \cap \bar{L}_{k}$, и, следовательно, множество $\mathscr{S} n$-кратно покрывает точку $w=0(\mathrm{c}$ учетом кратности). Здесь $\bar{L}_{k}$ означает замыкание $L_{k}$ в $\mathscr{R}$. Заметим, что для любого $k, 1 \leqslant k \leqslant n$, множество $\mathscr{S} \cap \bar{L}_{k}$ связно. Склеивая данный лист $L_{k^{\prime}}$ по одному из берегов разреза с другим листом $L_{k^{\prime \prime}}$ так, как это было на поверхности $\mathscr{R}$, получаем связное множество $\mathscr{S} \cap\left(\bar{L}_{k^{\prime}} \cup \bar{L}_{k^{\prime \prime}}\right)$. Затем к множеству $\bar{L}_{k^{\prime}} \cup \bar{L}_{k^{\prime \prime}}$ приклеиваем другой лист $L_{k^{\prime \prime \prime}}$, получая связное множество $\mathscr{S} \cap\left(\bar{L}_{k^{\prime}} \cup \bar{L}_{k^{\prime \prime}} \cup \bar{L}_{k^{\prime \prime \prime}}\right)$ и так далее, пока 
склеенные листы не образуют всю поверхность $\mathscr{R}$. Отсюда вытекает связность множества $\mathscr{S}$ и, как следствие, односвязность области $\mathscr{R} \backslash \mathscr{S}$. Обозначим через $f$ одну из регулярных ветвей аналитической функции $\omega=(P(z))^{1 / n}$ в односвязной области $B:=\mathscr{P}(\mathscr{R} \backslash \mathscr{S})$. Функция $f$ конформно и однолистно отображает область $B$ на область $f(B)$, представляющую собой $\omega$-плоскость, разрезанную по прямолинейным отрезкам. Каждый такой отрезок соединяет точку $\omega=0$ с точкой, модуль которой больше нуля, но не превосходит единицы. Число указанных отрезков не превосходит $n+m-1$. Действительно, каждая точка ветвления порождает $n_{\nu}+1$ отрезок, где $n_{\nu}-$ кратность нуля $\zeta_{\nu}$ производной полинома $P$, и некоторые из этих отрезков вырожденные, а

$$
\sum_{\nu=1}^{m}\left(n_{\nu}+1\right)=n-1+m .
$$

Отсюда следует, что континуум

$$
\Gamma=\left(\mathbb{C}_{\omega} \backslash f(B)\right) \cup\left(\bigcup_{j=1}^{n-m+1}\left[0, f\left(a_{j}\right)\right]\right)
$$

состоит из не более, чем $2 n$ невырожденных отрезков, выходящих из начала, и длины, не превосходящей единицы. Введем также симметричный континуум

$$
\Gamma^{*}=\left\{\omega:|\omega| \leqslant 1, \arg \omega^{2 n}=0\right\},
$$

состоящий из $2 n$ отрезков. Емкость сар $\Gamma^{*}$ равна $2^{-1 / n}[3 ;$ с. 216]. Из монотонности емкости и следствия 1.4 работы [6] имеем

$$
2^{-1 / n}=\operatorname{cap} \Gamma^{*} \geqslant \operatorname{cap} \Gamma .
$$

Осталось рассмотреть континуум

$$
\gamma=\mathscr{P}(\mathscr{S}) \cup f^{-1}\left(\bigcup_{j=1}^{n-m+1}\left[0, f\left(a_{j}\right)\right]\right),
$$

соединяющий точки $a_{j}, j=1, \ldots, n-m+1$, а также все нули и критические точки полинома $P$. Поскольку

$$
f\left(\overline{\mathbb{C}}_{z} \backslash \gamma\right)=\overline{\mathbb{C}}_{\omega} \backslash \Gamma
$$

и разложение функции $f$ в окрестности бесконечности имеет вид

$$
f(z)=\alpha z+\cdots, \quad|\alpha|=1,
$$

то

$$
\operatorname{cap} \Gamma=\operatorname{cap} \gamma
$$

Теорема доказана.

3. О континуумах наименьшей емкости. В теории функций хорошо известна задача Н. Г. Чеботарева о континууме $\gamma\left(c_{1}, \ldots, c_{l}\right)$ наименьшей емкости, содержащем заданные точки $c_{1}, \ldots, c_{l}$ плоскости $\mathbb{C}_{z}[7 ; \S 4,5]$. С этой задачей связаны многие экстремальные вопросы теории однолистных функций. Вместе с тем, ее решение при $n \geqslant 4$ до сих пор неизвестно. Даже в случае решения задачи Чеботарева емкость экстремального континуума наверняка будет иметь сложное аналитическое представление. Неравенство (3) позволяет оценить сар $\gamma\left(c_{1}, \ldots, c_{l}\right)$ сверху в ряде частных случаев. Рассмотрим некоторые из них. 
СледСТвиЕ 1. Для заданных точек $z_{\mu} \in \mathbb{C}_{z}, \mu=1, \ldots, n$, обозначим через $M$ величину $\max \left\{|P(\zeta)|: P^{\prime}(\zeta)=0\right\}$, где $P$ - полином (1). Тогда для любых двух точек $a_{1}$ и $a_{2}$, удовлетворяющих условию $\left|P\left(a_{j}\right)\right| \leqslant M, j=1,2$, справедливо неравенство

$$
\operatorname{cap} \gamma\left(z_{1}, \ldots, z_{n}, a_{1}, a_{2}\right) \leqslant\left(\frac{M}{2}\right)^{1 / n} .
$$

Равенство в (4) достигается, например, в случае, когда $z_{\mu} 2^{1 / n-1}, \mu=1, \ldots, n,-$ нули полинома Чебъшева $T_{n}(z), u a_{j}=(-1)^{j} 2^{1-1 / n}, j=1,2$.

ДокАЗАТЕЛЬСТво. Полином $S(z)=P\left(M^{1 / n} z\right) / M=z^{n}+\cdots$ удовлетворяет условию (2):

$$
\max \left\{|S(\zeta)|: S^{\prime}(\zeta)=0\right\}=\max \left\{|P(\zeta)| / M: P^{\prime}(\zeta)=0\right\}=1 .
$$

Точки $z_{\mu} M^{-1 / n}, \mu=1, \ldots, n$, являются нулями полинома $S$ и, кроме того, $\left|S\left(a_{j} M^{-1 / n}\right)\right| \leqslant 1, j=1,2$. По теореме существует континуум $\gamma$ емкости

$$
\operatorname{cap} \gamma \leqslant 2^{-1 / n}
$$

проходящий через точки $z_{\mu} M^{-1 / n}, \mu=1, \ldots, n$, и $a_{j} M^{-1 / n}, j=1,2$. Поэтому континуум $\Gamma:=\left\{z: z M^{-1 / n} \in \gamma\right\}$ соединяет точки $z_{\mu}, \mu=1, \ldots, n$, и $a_{j}, j=1,2$, и выполняются неравенства

$$
\operatorname{cap} \gamma\left(z_{1}, \ldots, z_{n}, a_{1}, a_{2}\right) \leqslant \operatorname{cap} \Gamma \leqslant(M / 2)^{1 / n} .
$$

Случай равенства в (4) проверяется непосредственно. Следствие доказано.

В связи с полученным неравенством представляется интересным установить оценку сверху для величины $\max \left\{|P(\zeta)|: P^{\prime}(\zeta)=0\right\}$ в случае, когда все нули $z_{\mu}, \mu=$ $1, \ldots, n$, полинома $P(z)=z^{n}+\cdots$ лежат в некотором круге $|z| \leqslant R$. Заметим, что в этом случае из свойств диссимметризации [6] легко следует оценка

$$
\operatorname{cap} \gamma\left(z_{1}, \ldots, z_{n}\right) \leqslant 2^{-2 / n} R
$$

с равенством для точек $z_{\mu}^{*}=R \exp (2 \pi i \mu / n), \mu=1, \ldots, n ; \gamma\left(z_{1}^{*}, \ldots, z_{n}^{*}\right)=\{z:|z| \leqslant$ $\left.R, \arg z^{n}=0\right\}$.

Широта применения следствия 1 ограничена сложностью вычисления константы $M$. Однако доказанная выше теорема дает также другой подход к оценкам наименьшей емкости.

СлеДСтвиЕ 2. Для заданных точек $\zeta_{\nu} \in \mathbb{C}_{z}, \nu=1, \ldots, n-1$, пусть

$$
P(z)=n \int_{0}^{z}\left(z-\zeta_{1}\right) \ldots\left(z-\zeta_{n-1}\right) d z+d_{0}=z^{n}+\cdots,
$$

где $d_{0}$ - некоторое комплексное число. Тогда для любых точек $a_{1}$ и $a_{2}$, удовлетворяющих условию

$$
\left|P\left(a_{j}\right)\right| \leqslant M:=\max _{1 \leqslant \nu \leqslant n-1}\left|P\left(\zeta_{\nu}\right)\right|,
$$

справедливо неравенство

$$
\operatorname{cap} \gamma\left(\zeta_{1}, \ldots, \zeta_{n-1}, a_{1}, a_{2}\right) \leqslant(M / 2)^{1 / n} .
$$

Знак равенства имеет место, например, в случае, когда $\zeta_{\nu} 2^{1 / n-1}, \nu=1, \ldots, n-1,-$ нули производной полинома Чебышева $T_{n}(z), d_{0}=T_{n}(0)$ и $a_{j}=(-1)^{j} 2^{1-1 / n}, j=1,2$. 
ДокАЗАТЕЛьСтво этого утверждения повторяет с небольшими изменениями доказательство следствия 1. Необходимо лишь учесть прохождение континуума $\gamma$ через критические точки подходящего полинома.

В качестве примера, положим в следствии $2 \zeta_{1}=-1, \zeta_{2}=1$, и $d_{0}=0$. Тогда

$$
\gamma\left(-1,1, a_{1}, a_{2}\right) \leqslant 1
$$

для любых точек $a_{1}$ и $a_{2}$ из лемнискаты $\left|z^{3}-3 z\right| \leqslant 2$ с равенством в случае $a_{1}=-2$ и $a_{2}=2$.

Следующий результат не требует пояснений.

СлеДСтвиЕ 3. Для любых точек $a_{j}, j=1, \ldots, n$, принадлежсащих лемнискате

$$
\left|z^{n}-1\right| \leqslant 1
$$

справедливо неравенство

$$
\operatorname{cap} \gamma\left(0, \eta_{1}, \ldots, \eta_{n}, a_{1}, \ldots, a_{n}\right) \leqslant 2^{-1 / n},
$$

где $\eta_{j}, j=1, \ldots, n,-к о р н и ~ n-\check{~ с т е п е н и ~ и з ~ е д и н и ц ъ . ~ Р а в е н с т в о ~ д о с т и г а е т с я ~ д л я ~}$ mочек $a_{j}=\sqrt[n]{2} \eta_{j}, j=1, \ldots, n$.

В частном случае, при $n=2$, из следствия 3 вытекает, что для любых точек $z_{1}, z_{2} \in \mathbb{C}_{z}$ и любых точек $a_{1}, a_{2}$, принадлежащих лемнискате

$$
\left|\left(z-z_{1}\right)\left(z-z_{2}\right)\right| \leqslant \frac{\left|z_{1}-z_{2}\right|^{2}}{4},
$$

справедливы неравенства

$$
\frac{\left|z_{1}-z_{2}\right|}{4} \leqslant \operatorname{cap} \gamma\left(z_{1}, z_{2}, \frac{\left(z_{1}+z_{2}\right)}{2}, a_{1}, a_{2}\right) \leqslant \frac{\left|z_{1}-z_{2}\right|}{2 \sqrt{2}} .
$$

Знак равенства в правом неравенстве имеет место в случае $a_{1}=z_{2}+\left(z_{1}-z_{2}\right)(1+\sqrt{2}) / 2$ и $a_{2}=z_{1}+\left(z_{2}-z_{1}\right)(1+\sqrt{2}) / 2$. Левое неравенство классическое и приводится лишь для сравнения.

Автор выражает благодарность Л. В. Ковалеву за полезные замечания к данной работе.

\section{СПИСОК ЦИТИРОВАННОЙ ЛИТЕРАТУРЫ}

[1] P. Borwein, T. Erdelyi, "Polynomials and polynomial inequalities", Grad. Texts in Math., 161, Springer-Verlag, New York, 1995.

[2] Q. I. Rahman, G. Schmeisser, "Analytic Theory of Polynomials", London Math. Soc. Monographs, New Series, 26, Clarendon Press, Oxford, 2002.

[3] Н. С. Ландкоф, Основы современной теории потенциала, Наука, М., 1966.

[4] G. Polya, "Beitrag zur Verallgemeinerung des Verzerrungssatzes auf mehrfach Zusammenhängende Gebiete", S.B. Preuss. Akad. Wiss., K.L. Math. Phys. Tech., Berlin, 1928, 228-232.

[5] С. Стоилов, Теория функций комплексного переменного, m. 1, Изд-во ИЛ, М., 1962.

[6] В.Н. Дубинин, "Симметризация в геометрической теории функций комплексного переменного", УМН, 49:1 (1994), 3-76.

[7] Г. В. Кузьмина, "Методы геометрической теории функций”, Алгебра и анализ, 9:3 (1997), 41-103; № 5, 1-50.

В.Н. Дубинин

Институт прикладной математики ДВО РАН

E-mail: dubinin@iam.dvo.ru
Поступила в редакцию

01.12 .2005 